\title{
A preliminary presentation of a mobile co-operative platform for Heart Failure self-management
}

\author{
Georgia S. Karanasiou, Fanis G. Kalatzis, Evanthia E. Tripoliti, Abdelhamid Errachid, Maria \\ Giovanna Trivella, Roger Fuoco, Fabio Di Francesco, Alicia Martínez-García, Carlos Luís Parra- \\ Calderón, Jochen K Schubert, Wolfram Miekisch, Joan R. Bausells, Senior Member, IEEE, Themis P. \\ Exarchos, Dimitrios I. Fotiadis, Senior Member, IEEE
}

\begin{abstract}
Heart Failure (HF) is a rapidly increasing cardiovascular chronic disease that affects millions of people globally. Lack of proper management of HF patients increases the risk of frailty and other undesirable effects and contributes to loss of independence. The engagement of the HF patient and all actors related to his/her disease management is critical for empowering the patients in achieving sustainable behaviour change, regarding their adherence and compliance. To address this, the concept and the architecture of a mobile co-operative platform are described. The design and development is based on a multi-stakeholder patient centered mHealth ecosystem for HF patients that will facilitate the collaboration of multidisciplinary actors.
\end{abstract}

\section{INTRODUCTION}

$\mathrm{H}$ eart Failure (HF) is a debilitating and long-term condition which affects 26 million people globally,

The work of the authors was supported by the HEARTEN project that has received funding from the European Union's Horizon 2020 research and innovation programme under grant agreement No 643694.

G.S. Karanasiou, F.G. Kalatzis and E.E Tripoliti are with the Department of Biomedical Research, Institute of Molecular Biology and Biotechnology, FORTH, Ioannina, (phone: 30-26510-07702, e-mail: g.karanasiou@gmail.com, tkalatz@gmail.com, etripoliti@gmail.com)

A. Errachid. is with the Université de Lyon, Institut de Sciences Analytiques (ISA) - UMR 5280, 5 rue de la Doua, 69100 Villeurbanne, France (e-mail: abdelhamid.errachid@univ-lyon1.fr)

M.G. Trivella is with the CNR Institute of Clinical Physiology and University of Pisa, Pisa, Italy (e-mail: trivella@ifc.cnr.it)

R. Fuoco and F.Di Francesco are with the Department of Chemistry and Indurstrial Chemistry, University of Pisa, Pisa, Italy (email: roger.fuoco@unipi.it, fabio.difrancesco@unipi.it)

M. Marzilli is with the Azienda Ospedaliera-Universitaria Pisana, Cardiothoracic and Vascular Department, Pisa, Italy (email: mario.marzilli@med.unipi.it)

A. Martínez-García, C.L. Parra-Calderón are with the Hospital Universitario Virgen Macarena - Virgen del Rocío, Sevilla, España (email: alicia.martinez.exts@juntadeandalucia.es,carlos.parra.sspa@juntadeandaluc ia.es)

J.K Schubert, W. Miekisch are with the Department of Anaesthesia and Intensive Care Medicine, University Medical Center Rostock, Rostock, Germany (email: jochen.schubert@uni-rostock.de, wolfram.miekisch@unirostock.de)

J.R. Bausells is with the Instituto de Microelectrónica de Barcelona, IMB-CNM (CSIC), Campus UAB, 08193 Bellaterra, Spain (email: joan.bausells@imb-cnm.csic.es)

D.I. Fotiadis is with the Department of Biomedical Research, Institute of Molecular Biology and Biotechnology, FORTH, Ioannina, Greece and Department of Materials Science, Unit of Medical Technology and Intelligent Information Systems, Ioannina, Greece (e-mail: fotiadis@cc.uoi.gr)

T.P. Exarchos is with the Institute of Communication and Computer Systems, Athens, Greece (email: themis.exarchos@gmail.com). while 3.6 million people are diagnosed with HF, every year [1]. Over the next 50 years, the number of people aged 65+, in the European Union (EU) is expected to almost double, from 85 million (2008) to 151 million (2060) [2]. Due to the fact that HF occurs mostly among elderly individuals, the increase of the life expectancy is expected to be followed by a progressive increase of HF incidence that will augment the challenge of appropriately and effectively managing the HF patients. In addition, the increasing incidence of HF poses a considerable economic burden; the annual cost of HF to insurers has been recently estimated to be approximately $6,000 €$ per person per year, and the cost related to hospitalization is more than $9,000 €$ [3]. The only way of decreasing this economic pandemic is by controlling the HF risk factors and managing more effectively the HF patients. HF patients are fortunate to have at their disposal a plethora of guidelines and health-based knowledge; however, the integration of this knowledge in their life becomes difficult. HF patients are among the most medication non adherent patients when compared to the patients with other chronic diseases [4]. Medication non adherence is a major concern and frequent issue with a negative impact in the patient's clinical condition, autonomy, physical and mental health and generally in their quality of life [5], [6]. Apart from medication adherence, there are additional factors that influence the success of the HF management such as nutrition [7], [8] physical activity [9], weight [10] and heart activity [11], [12].

Several studies have tried to approach HF disease through a variety of ways. SensorART provided a telemedicine service that supports HF patients and allows them to keep control of the performance of cardiovascular implanted assist devices and to be treated at home [13]. HeartCycle includes a closed-loop disease management solution that is achieved by multi-parametric monitoring, examination and analysis of vital signs, and other measurements to serve both HF and Coronary Heart Disease (CHD) patients [14]. In addition, the HeartWays system proposed home-based rehabilitation as an alternative to hospitalization, through developing wearable sensors and intelligent algorithms that would assist in HF patient follow-up and management [15]. VPH2 approached HF through patient-specific computational modeling in order to assist the cardiologists and the cardiac surgeons in clinical decision making and 
planning of the optimal treatment for patients with postischemic Left Ventricular Dysfunction (LVD), with or without ischemic mitral regurgitation (IMR) [16]. However, none of these systems addresses HF management in a holistic approach.

HEARTEN provides a complete end-to-end mHealth solution for HF patients. The target population of HEARTEN are patients with chronic and acute HF [17], either post-ischemic or with dilated cardiomyopathy [18], requiring occasional re-admittance into hospitals. The main idea is to develop biosensors that detect and quantify novel saliva and breath HF biomarkers, being identified through analytical techniques. The biomarkers reflect the health status of the HF patient and identify whether the patient is adherent to the administered drugs. The saliva biosensor will be integrated into the patient's cup, while the breath biosensor will be integrated into the smartphone. In addition, heart rate, blood pressure, respiration, body temperature, and physical activity sensors constitute the sensor kit of the patient. These sensor input data are complemented with: (i) nutrition information from the patient's/caregiver's smartphone, (ii) weight monitoring information through wireless weight scales and, (iii) patient's health record profile and information added by the caregivers and the healthcare professionals. The health data will be the input to a knowledge management system, in a cloud based reference architecture, that will deliver to all the ecosystem actors critical information, provide health support, and education to the HF patients.

The main scientific and technological objectives that are followed for the development of the HEARTEN system are:

- Continuous monitoring of specific biomarkers in breath and saliva in patients suffering from HF.

- Continuous monitoring of vital signals and measurements in patients suffering from HF.

- Development of mHealth applications for the patient and the ecosystem actors.

- Identification of trends and patterns of non-adherence through knowledge management systems.

- Development of the Information and Communication Technology (ICT) co-operative environment.

- Education and guidance of the ecosystem actors.

- Creation of a supportive and interactive ecosystem for holistic HF management.

\section{MATERIALS AND METHODS}

\section{A. Overall architecture}

The HEARTEN overall architecture is presented in Fig. 1. Through the mHealth application all the data from the sensors and other patient related data, will be gathered and transmitted to the HEARTEN cloud infrastructure, where the data mining and analytics will be implemented to provide critical information to all ecosystem actors.

Specifically, the main components of HEARTEN system are: (i) the sensors, (ii) the mHealth application, (iii) the databases, (iv) the Knowledge Management System (KMS), (v) the Dynamic Patient Communication Protocol (DynPCP) [19] platform, and (vi) the cloud platform. All sensor and patient related data will be sent to the mobile application of the user. HEARTEN design includes two different types of data: (i) Personal Data which are related to the users (patients, doctors, caregivers, etc.) that permit to build a registry of all the users and, (ii) Sensors Data. For these data two different types of databases are needed: (i) Relational Database based on MySQL for all the Personal Data and (ii) NoSQL Database based on mongoDB for all the unstructured raw data retrieved from the sensors and parsed from the applications (raw and processed data will be stored in different collections). The data are transmitted to the KMS where the data mining and the analytics will be implemented to extract rules and provide patterns regarding biomarker detection/medication intake, physical activity, nutrition, blood pressure and heart rate measurements. The DynPCP based on the different patient communication protocols (European Society of Cardiology guidelines [20]), the outcomes of the KMS and the patient's medical data will send notifications to the corresponding actors of HEARTEN environment. The notifications are initially sent to the mobile application of HEARTEN while DynPCP monitors the delivery and read status. In case of critical notifications, when immediate action is needed and the recipient does not receive or read the notification within a specified threshold, DynPCP also sends an SMS and in parallel informs all the other actors. DynPCP will monitor the results of the intervention tools by assessing the response time and the action which is taken by the patient, caregiver and healthcare professional based on an integrated evaluation algorithm.

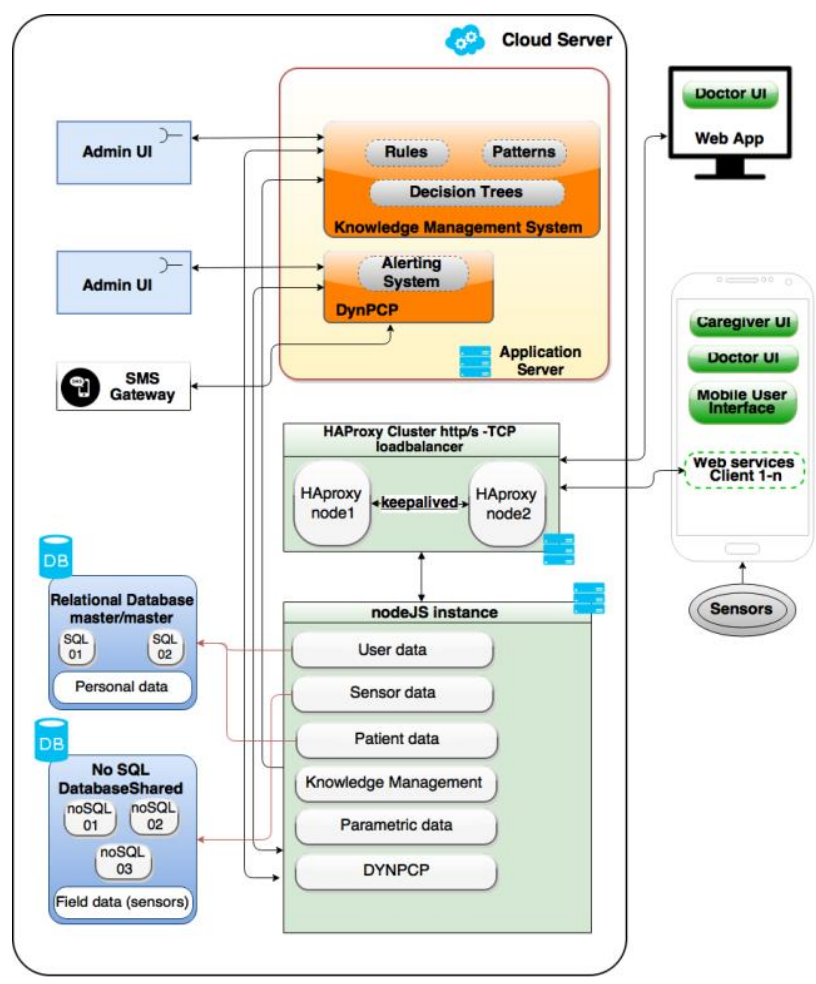

Fig.1. The overall HEARTEN architecture. 
The overall HEARTEN architecture is defined and designed with special emphasis on the function, standards, technical specifications, communication and dependencies of each of these components.

\section{B. Breath and Saliva sensors}

The final HEARTEN integrated solution goes beyond state of the art of existing patient self-management tools by developing breath and saliva sensors. To accomplish this, analytical techniques such as colorimetric or spectrophotometric methods, gas- or coupled with mass spectrometry, or capillary electrophoresis with UV detection are utilized for the saliva biomarkers detection [21], [22] and hyphenated analytical methodology based on thermal desorption, gas chromatography, and mass spectrometry (TD-GC-MS) are applied to define the HF-related biomarkers present in exhaled breath samples [23].

\section{Data Collection}

For the implementation of data mining approach and the discovery of interesting and useful patterns of patients' adherence, data from retrospective and prospective patients will be utilized. These data will be gathered from Universita di Pisa (Italy) and Servicio Andaluz de Salud (Spain). The enrolled patients will be asked to use the sensors in a home environment. The annotated data that will be gathered include:

- Measurements of breath and saliva biomarkers from the sensors.

- Measurements from the heart rate, respiration, weight scale, blood pressure, activity, and body temperature sensors.

More specifically, data gathering will consist of four phases. In the $1^{\text {st }}$ phase, retrospective data from HF patients will be used. In the $2^{\text {nd }}$ and $3^{\text {rd }}$ phase, prospective data from HF patients will be collected that will include measurements of heart rate, respiration, weight scale, blood pressure, activity, and body temperature while in the $4^{\text {th }}$ phase, additional data from breath and saliva sensors will be collected.

\section{Data Mining}

HEARTEN system will address all the issues that HF patients experience through efficiently and effectively monitoring their status and providing patterns and knowledge extraction. Several data mining techniques will be customized and stored into the cloud for analysis of the data of the patients in two phases [24]. In the first phase, the data of the patients will be used for creating the data mining structures, developing and training the predictive models, whereas based in the grouping of patients, already defined models will be utilized for providing trends of HF patients' non adherence. More specifically, the everyday activities of a patient will be examined and analyzed so as to develop the personalized profile of adherence.

In parallel, patients that have similar disease and lifestyle characteristics, receive the same treatment or have the same adherence behaviour will be used for developing the predictive models for alert provisioning and decision support for the patients, the caregivers and the healthcare professionals.

\section{E. mhealth Apps and decision support}

HEARTEN will develop a mHealth application by adopting imperative design characteristics for involving and motivating the patient in self-management and cultivate positive behaviour towards HF. More specifically the mobile application design and development will follow the Goal Oriented Design Methodology, which is a user-centered approach, commonly utilized in contemporary design and particularly in consumer -oriented products, that especially fits for the $\mathrm{HF}$ patients by empowering them to reach specific HF management objectives and goals (monitoring of medication, blood pressure, heart rate, physical activity, and nutrition) [25].

Specific attention is paid in the design approach based on the following requirements: (i) accommodate patients with different health profiles (HF severity, sex, age and lifestyle), (ii) accommodate different users (patient, caregiver and healthcare professional), (iii) design based on user needs' identification, (iv) design based on the patient's lifestyle, (v) user friendliness (user interface, least amount of learning requirements), (vi) user satisfaction and (vii) communication between the ecosystem actors.

Specifically, through the mobile apps, the patient will be able to: (i) import his/her daily nutrition, (ii) preview his/her health status based on the measurements of the sensors, (iii) receive notifications and alerts from the caregiver/healthcare professional, (iv) send a direct message to the caregiver/healthcare professional, and (v) be educated about HF. An open dialogue and communication with the caregiver and the healthcare professional will prevent critical clinical situations and co-morbidities, such as renal dysfunction, cerebral dysfunction, anaemia, liver dysfunction, chronic obstructive pulmonary disease, and diabetes.

In addition, this direct way of patient empowerment will not only help to avoid critical situations but also support and educate the patient to reduce conditions leading to such situations and, therefore, support health education and selfmanagement in general.

\section{F. Ecosystem}

HEARTEN solution is the first mHealth system for monitoring and managing patients suffering from HF. HEARTEN engages the whole HF healthcare ecosystem as presented in Fig. 2.

The involvement of the patients does not only concern the availability of information or health literacy, but mainly their interaction with the caregivers, the health professionals and other ecosystem actors. The HF patients do not just want more information; they are in need of an open line communication, including all actors of the ecosystem, enabling the management of all the HF related issues, including medication, physical activity and nutrition 
adherence.

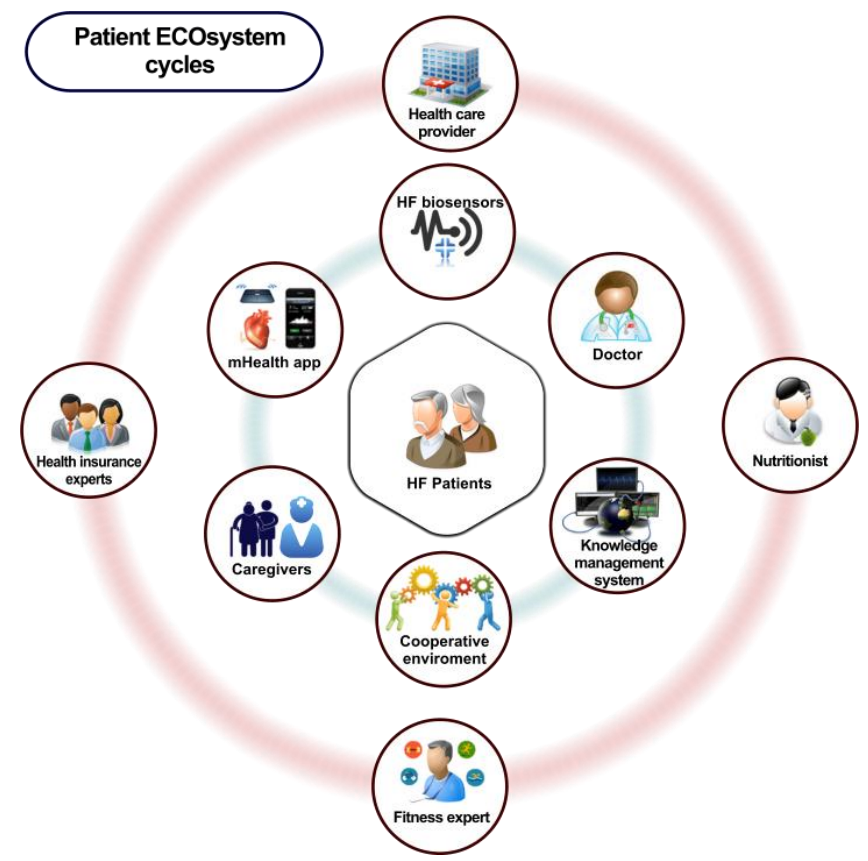

Fig.2. The patient-centric approach of the HEARTEN ecosystem.

\section{CONCLUSIONS}

Through HEARTEN the HF patients are empowered in self-management, by using their smartphones and tracking their medical vital signs, while the healthcare professionals and the caregivers can provide warnings, coordinate therapies, improve adherence, and intervene before frailty incidences occur.

The traditional medical knowledge and the decision support methods in tandem with mobile and cloud infrastructure will result in a patient-centered ecosystem that will make the HF patients active, involved, informed, educated, concerned, and empowered in HF management. Qualitative and quantitative evidence of the clinical outcomes combined with economic evaluation will provide valuable information for the gained benefits and the costs of HEARTEN solution.

\section{REFERENCES}

[1] A. S. Go, D. Mozaffarian, V. L. Roger, E. J. Benjamin, J. D. Berry, W. B. Borden, D. M. Bravata, S. Dai, E. S. Ford, C. S. Fox, S. Franco, H. J. Fullerton, C. Gillespie, S. M. Hailpern, J. A. Heit, V. J. Howard, M. D. Huffman, B. M. Kissela, S. J. Kittner, D. T. Lackland, J. H. Lichtman, L. D. Lisabeth, D. Magid, G. M. Marcus, A. Marelli, D. B. Matchar, D. K. McGuire, E. R. Mohler, C. S. Moy, M. E. Mussolino, G. Nichol, N. P. Paynter, P. J. Schreiner, P. D. Sorlie, J. Stein, T. N. Turan, S. S. Virani, N. D. Wong, D. Woo, M. B. Turner, and American Heart Association Statistics Committee and Stroke Statistics Subcommittee, "Heart disease and stroke statistics--2013 update: a report from the American Heart Association," Circulation, vol. 127, no. 1, pp. e6-e245, Jan. 2013.

[2] N. Reynolds and M. Turcsányi-Szabó, Key Competencies in the Knowledge Society: IFIP TC 3 International Conference, KCKS 2010, Held as Part of WCC 2010, 2010.

[3] M. R. Cowie, "The heart failure epidemic," Medicographia, 2012.

[4] N. Cottrell, Denaro, and L. Emmerton, "Exploring beliefs about heart failure treatment in adherent and nonadherent patients: use of the repertory grid technique," $P P A$, vol. Volume 7, pp. 141-150, Feb. 2013.

[5] M. Chisholm-Burns and C. Spivey, "The cost of medication nonadherence: consequences we cannot afford to accept.," $J \mathrm{Am}$ Pharm Assoc, vol. 52, pp. 823-826, 2003.

[6] A. A. Fitzgerald, J. D. Powers, P. M. Ho, T. M. Maddox, P. N. Peterson, L. A. Allen, F. A. Masoudi, D. J. Magid, and E. P. Havranek, "Impact of medication nonadherence on hospitalizations and mortality in heart failure," J. Card. Fail., vol. 17, no. 8, pp. 664-669, Aug. 2011.

[7] D. Gupta, V. V. Georgiopoulou, A. P. Kalogeropoulos, S. B. Dunbar, C. M. Reilly, J. M. Sands, G. C. Fonarow, M. Jessup, M. Gheorghiade, C. Yancy, and J. Butler, "Dietary Sodium Intake in Heart Failure," Circulation, vol. 126, no. 4, pp. 479-485, Jul. 2012.

[8] E. Colin-Ramirez, L. Castillo-Martinez, A. Orea-Tejeda, Y. Zheng, C. M. Westerhout, and J. A. Ezekowitz, "Dietary fatty acids intake and mortality in patients with heart failure," Nutrition, vol. 30, no. 11-12, pp. 1366-1371, 2014.

[9] J.-Y. Tabet, P. Meurin, A. Ben Driss, H. Weber, N. Renaud, A. Grosdemouge, F. Beauvais, and A. Cohen-Solal, "Benefits of exercise training in chronic heart failure," Archives of Cardiovascular Diseases, vol. 102, no. 10, pp. 721-730, 2009.

[10] C. J. Lavie, M. A. Alpert, and H. O. Ventura, "Risks and Benefits of Weight Loss in Heart Failure," Heart Failure Clinics, vol. 11, no. 1, pp. $125-131,2015$

[11] T. T. Lee, J. Chen, D. J. Cohen, and L. Tsao, "The association between blood pressure and mortality in patients with heart failure," American Heart Journal, vol. 151, no. 1, pp. 76-83, 2006.

[12] M. Sadaka, A. Aboelela, S. Arab, and M. Nawar, "Electrocardiogram as prognostic and diagnostic parameter in follow up of patients with heart failure," Alexandria Journal of Medicine, vol. 49, no. 2, pp. 145-152, 2013.

[13] "SENSORART." [Online]. Available: http://www.sensorart.eu/.

[14] "HEARTCYCLE." [Online]. Available: http://www.heartcycle.eu/.

[15] "HEARTWAYS." [Online]. Available: www.heartways.eu.

[16] "European Commission : CORDIS : Projects and Results : Virtual pathological heart of the virtual physiological human." [Online]. Available: http://cordis.europa.eu/project/rcn/87317_en.html.

[17] S. M. Joseph, A. M. Cedars, G. A. Ewald, E. M. Geltman, and D. L. Mann, “Acute Decompensated Heart Failure," Tex Heart Inst J, vol. 36 , no. 6, pp. 510-520, 2009.

[18] "Dilated Cardiomyopathy (DCM)." [Online]. Available: http://www.heart.org/HEARTORG/Conditions/More/Cardiomyopat hy/Dilated-Cardiomyopathy_UCM_444187_Article.jsp. [Accessed: 14-Jul-2015].

[19] “Clinical Research.” [Online]. Available: /services/clinical-research. [Accessed: 16-Jul-2015].

[20] "Acute and Chronic Heart Failure." [Online]. Available: http://www.escardio.org/Guidelines-\&-Education/Clinical-PracticeGuidelines/Acute-and-Chronic-Heart-Failure. [Accessed: 14-Jul2015].

[21] R. Denver, A. Tzanidis, P. Martin, and H. Krum, "Salivary endothelin concentrations in the assessment of chronic heart failure," Lancet, vol. 355, no. 9202, pp. 468-469, Feb. 2000

[22] A. G. White, H. Gordon, and L. Leiter, "Studies in edema- the effect of congestive heart failure on saliva electrolyte concentrations," Journal of Clinical Investigation, vol. 29, no. 11, pp. $1445-1447,1950$

[23] T. Bhuiyan and M. S. Maurer, "Heart Failure with Preserved Ejection Fraction: Persistent Diagnosis, Therapeutic Enigma," Curr Cardiovasc Risk Rep, vol. 5, no. 5, pp. 440-449, Oct. 2011.

[24] L. Aleksovska-Stojkovska and S. Loskovska, Data Mining in Clinical Decision Support Systems. Springer Berlin Heidelberg, 2013.

[25] A. Paulo, Handbook of Research on Mobile Software Engineering: Design, Implementation, and Emergent Applications. IGI Global, 2012. 